Pacific Journal of Mathematic 


\section{BOUNDS FOR THE PERRON ROOT OF A NONNEGATIVE IRREDUCIBLE PARTITIONED MATRIX}

\section{Emeric Deutsch}

It is well-known that the Perron root of a nonnegative irreducible matrix lies between the smallest and the largest row sum of $A$. This result is generalized to the case when the matrix $A$ is partitioned into blocks.

1. Introduction and notations. If $A=\left(a_{i j}\right)$ is a nonnegative irreducible $n \times n$ matrix, then the Perron root $r(A)$ of $A$ satisfies the classical inequalities of Frobenius $[1$, p. $37 ; 9 ; 10$, p. $63 ; 21$, p. 31]

$$
\min _{i} S_{i} \leqq r(A) \leqq \max _{i} S_{i}
$$

where $S_{i}$ denotes the $i$ th row sum of $A$, i.e., $S_{i}=\sum_{y_{1}=1}^{n} a_{i j}(i=1, \cdots, n)$. Moreover, we have strict inequalities in (1) unless all the $S_{i}$ 's are equal.

Other bounds for $r(A)$ have been found by Ledermann [13], Ostrowski [15], Brauer [2], Ostrowski and Schneider [17], Hall and Porsching [11], Brauer and Gentry [3;4], and Deutsch [8]. (In some of these papers one has assumed that $A$ is a positive matrix.)

The purpose of this paper is to give some simple generalizations of the inequalities (1), by considering certain partitionings of $A$.

We introduce a few notations. By $\boldsymbol{R}^{m}$ we denote the vector space of all column $m$-tuples of real numbers and $(x)_{i}$ denotes the $i$ th (scalar) component of the vector $x \in \boldsymbol{R}^{m}$. By $\boldsymbol{R}^{m \times m}$ we denote the algebra of all $m \times m$ real matrices and $(A)_{i j}$ denotes the (scalar) $(i, j)$-entry of the matrix $A \in \boldsymbol{R}^{m \times m}$. For two vectors $x, y \in \boldsymbol{R}^{m}$, the inequality $x \leqq y(x<y)$ means $\left(x_{i}\right) \leqq(y)_{i}\left((x)_{i}<(y)_{i}\right)$ for all $i=1, \cdots, m$. If $X_{1}, \cdots, X_{t} \in \boldsymbol{R}^{m \times m}$, then $\Lambda_{s=1}^{t} X_{s}\left(\mathbf{V}_{s=1}^{t} X_{s}\right)$ denotes the greatest lower bound (least upper bound) of the matrices $X_{1}, \cdots, X_{t}$ in the natural (i.e., componentwise) partial ordering of $\boldsymbol{R}^{m \times m}$. In other words,

$$
\left(\bigwedge_{s=1}^{t} X_{s}\right)_{i j}=\min _{s=1, \cdots, t}\left(X_{s}\right)_{i j}, \quad\left(\bigvee_{s=1}^{t} X_{s}\right)_{i j}=\max _{s=1, \cdots, t}\left(X_{s}\right)_{i j},
$$

for all $i, j=1, \cdots, m$.

The transpose of a matrix $A$ (vector $u$ ) will be denoted by $A^{\top}$ $\left(u^{\top}\right)$ and the Perron root of a nonnegative matrix $A \in \boldsymbol{R}^{m \times m}$ will be denoted by $r(A)$.

2. Let 


$$
A=\left(\begin{array}{cccc}
A_{11} & A_{12} & \cdots & A_{1 k} \\
A_{21} & A_{22} & \cdots & A_{2 k} \\
\cdots & \cdots & \cdots & \cdots \\
A_{k 1} & A_{k 2} & \cdots & A_{k k}
\end{array}\right)
$$

be a nonnegative irreducible $n \times n$ matrix, where $A_{i j}$ is an $n_{i} \times n_{j}$ submatrix $(i, j=1, \cdots, k)$. Clearly, $n_{1}+\cdots+n_{k}=n$.

Let $p_{i j}$ denote the smallest row sum of $A_{i j}$, let $q_{i j}$ denote the largest row sum of $A_{i j}(i, j=1, \cdots, k)$ and consider the $k \times k$ matrices

$$
P(A)=\left(p_{i j}\right)_{i, j=1, \cdots, k}, \quad Q(A)=\left(q_{i j}\right)_{i, j=1, \cdots, k} .
$$

Proposition 1. We have

$$
r(P(A)) \leqq r(A) \leqq r(Q(A)) .
$$

Proof. Let $x \in \boldsymbol{R}^{n}$ be a Perron eigenvector of $A$, i.e.,

$$
A x=\rho x \quad(x>0),
$$

where $\rho=r(A)$. We partition $x$ as

$$
x=\left(\begin{array}{c}
x_{1} \\
\vdots \\
x_{k}
\end{array}\right) \in R^{n},
$$

where $x_{j} \in \boldsymbol{R}^{n_{j}}(j=1, \cdots, k)$. Now, equation (5) can be written

$$
A_{i 1} x_{1}+\cdots+A_{i k} x_{k}=\rho x_{i} \quad(i=1, \cdots, k) .
$$

We assume that $\left(x_{i}\right)_{M_{i}}$ is the smallest (scalar) component of $x_{i}$, i.e.,

$$
\left(x_{i}\right)_{M_{i}}=\min \left\{\left(x_{i}\right)_{1},\left(x_{i}\right)_{2}, \cdots,\left(x_{i}\right)_{n_{i}}\right\} .
$$

Equating the $M_{i}$ th components of both sides of (6), we obtain

$$
\rho\left(x_{i}\right)_{M_{i}}=\left(A_{i 1} x_{1}\right)_{M_{i}}+\cdots+\left(A_{i k} x_{k}\right)_{M_{i}},
$$

or

$$
\rho\left(x_{i}\right)_{M_{i}}=\sum_{s=1}^{n_{1}}\left(A_{i 1}\right)_{\boldsymbol{M}_{i}, s}\left(x_{1}\right)_{s}+\cdots+\sum_{s=1}^{n_{k}}\left(A_{i k}\right)_{\mathcal{M}_{i}, s}\left(x_{k}\right)_{s},
$$

whence, replacing $\left(x_{j}\right)_{s}$ by $\left(x_{j}\right)_{M_{j}}$ and then replacing the row sums of $A_{i j}$ by $p_{i j}$, we have

$$
\rho\left(x_{i}\right)_{M_{i}} \geqq p_{i 1}\left(x_{1}\right)_{M_{1}}+\cdots+p_{i k}\left(x_{k}\right)_{M_{k}} \quad(i=1, \cdots, k) .
$$

Introducing the vector 


$$
v=\left(\left(x_{1}\right)_{\mathbb{M}_{1}}, \cdots,\left(x_{k}\right)_{\mathbb{M}_{k}}\right)^{\top} \in \boldsymbol{R}^{k},
$$

inequalities ( 7 ) can be written as

$$
\rho v \geqq P(A) v \quad(v>0),
$$

which implies $[1$, p. $28 ; 5 ; 22$, p. 33] $r(P(A)) \leqq \rho=r(A)$.

The right-hand inequality of (4) is proved in an entirely similar manner.

REMARK 1. Since $q_{i j}$ is the row-sum norm [20, p.180] of $A_{i j}$, the right-hand inequality of (4) follows at once also from the theory of matricial norms $[6 ; 7]$, (see also $[16 ; 18 ; 19]$ ).

Proposition 2. Either $P(A)=Q(A)$, or

$$
r(P(A)<r(A)<r(Q(A)) .
$$

Proof. Assume $P(A) \neq Q(A)$. We construct a nonnegative irreducible matrix $B \in \boldsymbol{R}^{n \times n}$ by decreasing certain entries of $A$ so that $P(B)=Q(B)=P(A)$. Then $r(B)<r(A)[1$, p. 27; 21, p. 30] and, by Proposition 1, $r(B)=r(P(B)$ ). Consequently, $r(P(A))<r(A)$. Similarly, we construct a nonnegative irreducible matrix $C \in \boldsymbol{R}^{n \times n}$ by increasing certain entries of $A$ so that $P(C)=Q(C)=Q(A)$. Then $r(A)<r(C)$ and, by Proposition 1, $r(C)=r(P(C))$. Consequently, $r(A)<r(Q(A))$.

CoROLlaRY 1. The following statements are equivalent:

(a) $P(A)=Q(A)$

(b) $r(A)=r(P(A))$;

(c) $r(A)=r(Q(A))$;

(d) $r(P(A))=r(Q(A))$.

REMARK 2. If a nonnegative irreducible matrix $A \in \boldsymbol{R}^{n \times n}$, partitioned as in (1), satisfies the equivalent conditions of Corollary 1 , then it follows from condition (a) that, for each fixed pair $i, j \in$ $\{1, \cdots, k\}$, all the row sums of $A_{i j}$ are equal to $p_{i j}\left(=q_{i j}\right)$. Thus, $A$ is a so-called block-stochastic matrix [12]. In this case, every eigenvalue of $P(A) \in \boldsymbol{R}^{k \times k}$ is an eigenvalue of $A \in \boldsymbol{R}^{n \times n}$ (see [12, Theorem 2]).

EXAMPLE 1. We consider the partitioned matrix

$$
A=\left(\begin{array}{ll:l}
1 & 1 & 2 \\
2 & 1 & 3 \\
\hdashline 2 & 3 & 5
\end{array}\right) .
$$


We have

$$
P(A)=\left(\begin{array}{ll}
2 & 2 \\
5 & 5
\end{array}\right), \quad Q(A)=\left(\begin{array}{ll}
3 & 3 \\
5 & 5
\end{array}\right),
$$

and $r(P(A))=7, r(Q(A))=8$. Thus, $7<r(A)<8$. This result is better than those obtained by several other methods $[4 ; 14$, p. 158].

EXAMPLE 2. We consider the partitioned matrix

$$
A=\left(\begin{array}{ccc:cc}
3 & 1 & 5 & 1 & 4 \\
2 & 2 & 5 & 2 & 3 \\
1 & 5 & 3 & 1 & 4 \\
\hdashline 1 & 1 & 3 & 4 & 1 \\
0 & 2 & 3 & 3 & 2
\end{array}\right) .
$$

We have

$$
P(A)=Q(A)=\left(\begin{array}{ll}
9 & 5 \\
5 & 5
\end{array}\right)
$$

and thus, in this case Proposition 1 yields the exact value of the Perron root of $A: r(A)=r(P(A))=r(Q(A))=7+\sqrt{29} \approx 12.38$. The matrix $A$ is block-stochastic (see Remark 2).

3. Let

$$
\begin{aligned}
& A=\left(\begin{array}{cccc}
A_{11} & A_{12} & \cdots & A_{1 N} \\
A_{21} & A_{22} & \cdots & A_{2 N} \\
\cdots & \cdots & \cdots & \cdots
\end{array}\right) \\
& A_{N 1} A_{N 2} \cdots A_{N N}
\end{aligned}
$$

be a nonnegative irreducible $n \times n$ matrix, where each $A_{i j}$ is a square $k \times k$ matrix. Clearly, $n=k N$.

Denote

$$
R_{i}(A)=\sum_{j=1}^{N} A_{i j} \in \boldsymbol{R}^{k \times k} \quad(i=1, \cdots, N) .
$$

Proposition 3. We have

$$
r\left(\bigwedge_{j=1}^{N} R_{j}(A)\right) \leqq r(A) \leqq r\left(\bigvee_{j=1}^{N} R_{j}(A)\right)
$$

Proof. Let $y \in \boldsymbol{R}^{n}$ be a Perron eigenvector of $G=A^{\top}$, i.e.,

$$
G y=\rho y \quad(y>0),
$$


where $\rho=r(A)$. We partition $y$ as

$$
y=\left(\begin{array}{c}
y_{1} \\
\vdots \\
y_{N}
\end{array}\right) \in \boldsymbol{R}^{n},
$$

where $y_{j} \in \boldsymbol{R}^{k}$ for all $j=1, \cdots, N$. Denoting $G_{i j}=A_{j i}^{\top}(i, j=1, \cdots, N)$, equation (11) can be written

$$
\sum_{j=1}^{N} G_{i j} y_{j}=\rho y_{i} \quad(i=1, \cdots, N) .
$$

Summing the equations (12) with respect to $i$, we obtain

$$
\sum_{i=1}^{N} \sum_{j=1}^{N} G_{i j} y_{j}=\rho w,
$$

where $w=\sum_{i=1}^{N} y_{i} \in \boldsymbol{R}^{k}$. Interchanging the order of summation in the left-hand side of (13), we have

$$
\rho w=\sum_{j=1}^{N}\left(R_{j}(A)\right)^{\top} y_{j},
$$

from where one has

$$
\left[\bigwedge_{j=1}^{N}\left(R_{j}(A)\right)^{\top}\right] w \leqq \rho w \leqq\left[\bigvee_{j=1}^{N}\left(R_{j}(A)\right)^{\top}\right] w .
$$

This, in turn, implies the inequalities $(10)[1$, p. 28; 5; 22, p. 33].

Proposition 4. Either $R_{1}(A)=\cdots=R_{N}(A)$, or

$$
r\left(\bigwedge_{j=1}^{N} R_{j}(A)\right)<r(A)<r\left(\bigvee_{j=1}^{N} R_{j}(A)\right) .
$$

Proof. Assume that $R_{1}(A), \cdots, R_{N}(A)$ are not equal. We construct a nonnegative irreducible $n \times n$ matrix $B$ by decreasing certain entries of $A$ so that $R_{1}(B)=\cdots=R_{N}(B)=\bigwedge_{j=1}^{N} R_{j}(A)$. Then $r(B)<$ $r(A)\left[1\right.$, p. 27; 21, p. 30] and, by Proposition 3, $r(B)=r\left(\bigwedge_{j=1}^{N} R_{j}(B)\right)$. Consequently, $r\left(\Lambda_{j=1}^{N} R_{j}(A)\right)<r(A)$. Similarly, we construct a nonnegative irreducible $n \times n$ matrix $C$ by increasing certain entries of $A$ so that $R_{1}(C)=\cdots=R_{N}(C)=\mathrm{V}_{j=1}^{N} R_{j}(A)$. Then $r(A)<r(C)$ and, by Proposition 3, $\quad r(C)=r\left(\bigvee_{j=1}^{N} R_{j}(C)\right)$. Consequently, $\quad r(A)<$ $r\left(\mathrm{~V}_{j=1}^{N} R_{j}(A)\right)$.

COROLlaRY 2. The following statements are equivalent:
(a) $R_{1}(A)=\cdots=R_{N}(A)$;
(b) $r(A)=r\left(\bigwedge_{j=1}^{N} R_{j}(A)\right)$;
(c) $r(A)=r\left(\bigvee_{j=1}^{N} R_{j}(A)\right)$; 
(d) $\quad r\left(\bigwedge_{j=1}^{N} R_{j}(A)\right)=r\left(\bigvee_{j=1}^{N} R_{j}(A)\right)$.

EXAMPLE 3. We consider the partitioned matrix

$$
A=\left(\begin{array}{ll:ll}
2 & 5 & 1 & 0 \\
0 & 0 & 1 & 2 \\
\hdashline 1 & 4 & 1 & 2 \\
1 & 1 & 0 & 1
\end{array}\right) .
$$

We have

$$
R_{1}(A)=\left(\begin{array}{ll}
3 & 5 \\
1 & 2
\end{array}\right), \quad R_{2}(A)=\left(\begin{array}{ll}
2 & 6 \\
1 & 2
\end{array}\right)
$$

and

$$
\bigwedge_{j=1}^{2} R_{j}(A)=\left(\begin{array}{ll}
2 & 5 \\
1 & 2
\end{array}\right), \quad \bigvee_{j=1}^{2} R_{j}(A)=\left(\begin{array}{ll}
3 & 6 \\
1 & 2
\end{array}\right)
$$

the last two matrices having Perron roots $2+\sqrt{5}$ and 5 , respectively. Thus, $4.236<r(A)<5$. The classical inequalities (1) yield only $3<r(A)<8$.

REMARK 3. The results of $\S 3$ can be obtained from those of $\S 2$. Indeed, if $A$ is the $n \times n$ matrix given in (8) and if we arrange the rows and columns of $A$ in the following positions;

$$
\begin{aligned}
& 1, N+1,2 N+1, \cdots,(k-1) N+1, \\
& 2, N+2,2 N+2, \cdots,(k-1) N+2, \\
& \cdots \cdots \cdots \cdots \cdots \cdots \cdots \cdots \cdots \cdots \cdots \\
& N, 2 N, 3 N, \cdots, k N
\end{aligned}
$$

then we obtain a matrix $A^{\prime}=\left(A_{i j}^{\prime}\right)_{i, j=1, \cdots, k} \in \boldsymbol{R}^{n \times n}$, where each $A_{i j}^{\prime}$ is an $N \times N$ submatrix. It can be easily seen that

$$
P\left(A^{\prime}\right)=\bigwedge_{i=1}^{N} R_{i}(A), \quad Q\left(A^{\prime}\right)=\bigvee_{i=1}^{N} R_{i}(A) .
$$

Since $r(A)=r\left(A^{\prime}\right)$, Propositions 3, 4 and Corollary 2 follow at once from Propositions 1, 2 and Corollary 1, respectively.

REMarK 4. It should be noted that the bounds given by Proposition 3 (or Proposition 1) are not always better than those given by the classical bounds (1). For example, considering the partitioned matrix 


$$
A=\left(\begin{array}{ll:ll}
3 & 0 & 0 & 1 \\
0 & 3 & 0 & 1 \\
\hdashline 1 & 1 & 1 & 1 \\
1 & 1 & 1 & 1
\end{array}\right)
$$

we have

$$
\begin{aligned}
& R_{1}(A)=\left(\begin{array}{ll}
3 & 1 \\
0 & 4
\end{array}\right), \quad R_{2}(A)=\left(\begin{array}{ll}
2 & 2 \\
2 & 2
\end{array}\right), \\
& \bigwedge_{j=1}^{2} R_{j}(A)=\left(\begin{array}{ll}
2 & 1 \\
0 & 2
\end{array}\right), \quad \bigvee_{j=1}^{2} R_{j}(A)=\left(\begin{array}{ll}
3 & 2 \\
2 & 4
\end{array}\right) \text {, }
\end{aligned}
$$

the last two matrices having Perron roots 2 and $\frac{1}{2}(7+\sqrt{17})$, respectively. Thus, $2<r(A)<5.562$. However, all row-sums of $A$ are equal to 4 and thus $r(A)=4$.

\section{REFERENCES}

1. A. Berman and R. J. Plemmons, Nonnegative Matrices in the Mathematical Sciences, Academic Press, New York, 1979.

2. A. Brauer, The theorems of Ledermann and Ostrowski on positive matrices, Duke Math. J., 24 (1957), 265-274.

3. A. Brauer and I. C. Gentry, Bounds for the greatest characteristic root of an irreducible nonnegative matrix, Linear Algebra and Appl., 8 (1974), 105-107.

4. - Bounds for the greatest characteristic root of an irreducible nonnegative matrix II, Linear Algebra and Appl., 13 (1976), 109-114.

5. L. Collatz, Einschliessungssatz für die charakteristischen Zahlen von Matrizen, Math. Z., 48 (1942), 221-226.

6. E. Deutsch, Matricial norms, Numer. Math., 16 (1970), 73-84.

7. - On matricial norms subordinate to vectorial norms, Math. Z., 122 (1971), $142-150$.

8. - Nested bounds for the Perron root of a nonnegative irreducible matrix, manuscript (see: Notices of the Amer. Math. Soc., 26 (1979), A 421-A 422).

9. G.F. Frobenius, Über Matrizen aus positiven Elementen, Sitzungsber. Königl. Preuss. Akad. Wiss. Berlin, (1908), 471-476.

10. F. R. Gantmacher, The Theory of Matrices, Vol. 2, Chelsea, New York, 1959.

11. C. A. Hall and T. A. Porsching, Bounds for the maximal eigenvalue of a nonnegative irreducible matrix, Duke Math. J., 36 (1969), 159-164.

12. E. V. Haynsworth, Applications of a theorem on partitioned matrices, J. Res. Nat. Bur. Standards, Sect. B, 62B (1959), 73-78.

13. W. Ledermann, Bounds for the greatest latent roots of a positive matrix, J. London Math. Soc., 25 (1950), 265-268.

14. M. Marcus and H. Minc, A Survey of Matrix Theory and Matrix Inequalities, Allyn and Bacon, Boston, 1964.

15. A. Ostrowski, Bounds for the greatest latent root of a positive matrix, J. London Math. Soc., 27 (1952), 253-256.

16. - On some metrical properties of operator matrices and matrices partitioned into blocks, J. Math. Anal. Appl., 2 (1961), 161-209.

17. A. Ostrowski and $\mathrm{H}$. Schneider, Bounds for the maximal characteristic root of a nonnegative irreducible matrix, Duke Math. J., 27 (1960), 547-553. 
18. F. Robert, Normes vectorielles de vecteurs et de matrices, Rev. Française Traitement Information (Chiffres), 7 (1964), 261-299.

19. _ - Sur les normes vectorielles régulières sur un espace vectoriel de dimension finie, C.R. Acad. Sci. Paris, 261 (1965), 5173-5176.

20. G. W. Stewart, Introduction to Matrix Computations, Academic Press, New York, 1973.

21. R. S. Varga, Matrix Iterative Analysis, Prentice-Hall, Englewood Cliffs, 1962.

22. H. Wielandt, Topics in the Analytic Theory of Matrices, Lecture notes prepared by Robert R. Meyer, Department of Mathematics, University of Wisconsin, Madison, 1967.

Received September 24, 1979.

Polytechnic Institute of New YoRK

BROOKLYN, NY 11201 


\section{PACIFIC JOURNAL OF MATHEMATICS}

\section{EDITORS}

DONALD BABBITT (Managing Editor)

University of Galifornia

Los Angeles, California 90024

Hugo RossI

University of Utah

Salt Lake City, UT 84112

C. C. MOORE AND ANDREW OGG

University of California

Berkeley, CA 94720
J. DUGUNDJI

Department of Mathematics University of Southern California Los Angeles, California 90007

R. Finn AND J. Milgram Stanford University Stanford, California 94305

\section{ASSOCIATE EDITORS}

R. ARENS

E. F. BECKENBACH

B. H. NEUManN

F. WOLF

K. YOSHIDA

\section{SUPPORTING INSTITUTIONS}

UNIVERSITY OF ARIZONA

UNIVERSITY OF BRITISH COLUMBIA CALIFORNIA INSTITUTE OF TECHNOLOGY

UNIVERSITY OF CALIFORNIA

MONTANA STATE UNIVERSITY

UNIVERSITY OF NEVADA, RENO

NEW MEXICO STATE UNIVERSITY

OREGON STATE UNIVERSITY
UNIVERSITY OF OREGON

UNIVERSITY OF SOUTHERN CALIFONIA

STANFORD UNIVERSITY

UNIVERSITY OF HAWAII

UNIVERSITY OF TOKYO

UNIVERSITY OF UTAH

WASHINGTON STATE UNIVERSITY

UNIVERSITY OF WASHINGTON 


\section{Pacific Journal of Mathematics}

\section{Vol. 92, No. $1 \quad$ January, 1981}

Michael E. Adams and J. Sichler, Lattices with unique complementation . ....1

Walter Allegretto, Positive solutions and spectral properties of second order

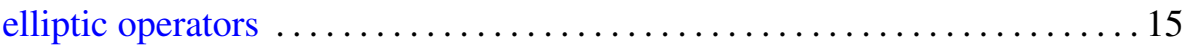

Philip J. Boland and Sean Dineen, Holomorphy on spaces of distribution . . 27

Duncan Alan Buell, Philip A. Leonard and Kenneth S. Williams, Note on

the quadratic character of a quadratic unit $\ldots \ldots \ldots \ldots \ldots \ldots \ldots \ldots \ldots$

Herbert Busemann and Bhalchandra B. Phadke, Two theorems on

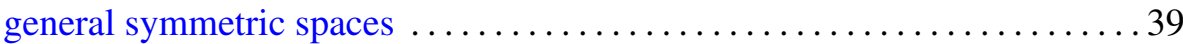

Emeric Deutsch, Bounds for the Perron root of a nonnegative irreducible

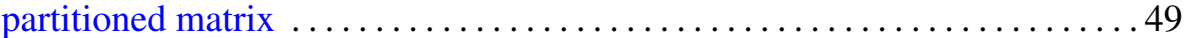

Charles F. Dunkl, A difference equation and Hahn polynomials in two

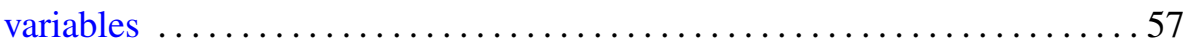

Gustave Adam Efroymson, The Riemann mapping theorem for planar Nash

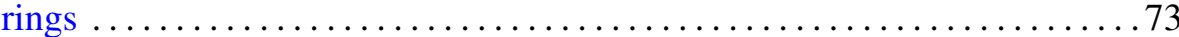

John Albert Fridy and Robert Ellis Powell, Tauberian theorems for matrices generated by analytic functions

Denton Elwood Hewgill, John Hamilton Reeder and Marvin Shinbrot,

Some exact solutions of the nonlinear problem of water waves .......887

Bessie Hershberger Kirkwood and Bernard Robert McDonald, The

symplectic group over a ring with one in its stable range

Esther Portnoy, Transitive groups of isometries on $H^{n}$

Jerry Ridenhour, On the sign of Green's functions for multipoint boundary

value problems

Nina M. Roy, An $M$-ideal characterization of $G$-spaces

Edward Barry Saff and Richard Steven Varga, On incomplete

polynomials. II

Takeyoshi Satō, The equations $\Delta u=P u(P \geqq 0)$ on Riemann surfaces and

isomorphisms between relative Hardy spaces

James Henry Schmerl, Correction to: "Peano models with many generic classes"

Charles Madison Stanton, On the closed ideals in $A(W)$

Viakalathur Shankar Sunder, Unitary equivalence to integral operators

Pavel G. Todorov, New explicit formulas for the $n$th derivative of composite functions

James Li-Ming Wang, Approximation by rational modules on boundary sets

Kenneth S. Williams, The class number of $Q(\sqrt{p})$ modulo 4 , for $p \equiv 5$

$(\bmod 8)$ a prime 